\title{
iPSCs, a Future Tool for Therapeutic Intervention in Mitochondrial Disorders: Pros and Cons
}

Mitochondrial disorders (MD) are multisystem diseases that may arise at any age, as a result of a dysfunction of the respiratory chain. The organs more frequently and severely affected are those that place high energetic demand like brain, skeletal muscle and heart. MD can be caused by mutations in the mitochondrial DNA (mtDNA) or in the nuclear genome (nDNA) (Wallace, 1999). In spite of the outstanding advances in the knowledge of the molecular basis of MD, the development of new therapies for these disorders is slow due in part to the lack of suitable disease models. Current therapies are predominantly focused on trying to alleviate symptoms, and there is an urgent need for the development of new therapeutic interventions. The potential of regenerative medicine has grown considerably in the last years since the Nobel Prize laureate Yamanaka reported that iPSCs could be generated from somatic cells by the ectopic expression of four transcription factors (Takahashi et al., 2007). iPSCs have major advantages including self-renewal and their capacity to differentiate into patientspecific cell lineages (Pareja-Galeano et al., 2016). In the last 4 years, iPSCs from patients with MD have been generated (Fujikura et al., 2012; Cherry et al., 2013; Folmes et al., 2013; H€am€al€ainen et al., 2013; Galera et al., 2016; Zurita et al., 2016; Zurita-Díaz et al., 2016). With this easily accessible source of cells, the possibilities are opened not only for regenerative medicine, but also for MD modeling and to screen potential new drugs. Regarding to this the generation of mutation-free iPSCs from MD patients could be an unlimited disease supply of cells for autologous transplantation therapy. Up to now, different strategies for achieving this are available. Among them, the new genome-editing techniques like the CRISPR/CAS9 system are very promising not only for correcting nDNA mutations but also for mutations in the mitochondrial genome (Jo et al., 2015). Other interesting possibilities are the generation of mutation free iPSCs through spontaneous segregation of heteroplasmic mtDNA in individual iPSCs lines or mitochondrial replacement by somatic cell nuclear transfer in MD caused by homoplasmic mtDNA mutations (Ma et al., 2015). However, an important issue remains before these iPSCs can be actually used in the clinical practice. This is mainly due to concerns that the epigenetic remodeling that occurs during the reprogramming process might originate genomic mutations that could make the cells tumorigenic. These concerns have led to different research studies related to the genomic integrity of the iPSCs but focusing only on a single type of genomic alteration and not considering the combined effects of single nucleotide variants (SNVs), copy number variations (CNVs) or structural variants. In this regard, Bhutani et al. (2016) assessed genome-wide mutation rates from replicate isogenic iPSC lines generated with the most commonly employed reprogramming methods (retrovirus, Sendai virus, and mRNA). Their interesting results show subtle differences in the number of identified variants depending on the reprogramming method and rarely mutations in genes associated with increased cancer risk. These data suggest that mutations previously reported by other groups in iPSCs are probably due to selective pressure that occurs when these cells are expanded in culture. In summary, based on the recent advances we highlight iPSCs as a promising tool for the study and development of new therapeutic approximations for MD. However, It should not be forgotten the importance of monitoring the appearance and consequences of mutations that can arise during the cell division and differentiation of the iPSCs. This way some of the concern about the theoretical chance that these transplanted cells may originate tumors or produce other pathologies could be more easily discarded.

Teresa Galera / Francisco Zurita-Díaz / Rafael Garesse / M. Esther Gallardo (1,2,3) 
1 Departamento de Bioquímica, Facultad de Medicina, Universidad Autónoma de Madrid, Madrid, Spain 2 Instituto de Investigaciones Biomédicas "Alberto Sols" UAM-CSIC and Centro de Investigación Biomédica en Red (CIBERER), Madrid, Spain

3 Instituto de Investigación Sanitaria Hospital 12 de Octubre (i+12), Madrid, Spain

Funded by:

-Centro de Investigación Biomédica en Red en enfermedades raras (CIBERER).

Grant Number: 13-717/132.05

-Instituto de Salud Carlos III cofunded by FEDER.

Grant Numbers: FIS PI10/0703, PI13/00556, PI15/00484

-Comunidad Autónoma de Madrid.

Grant Number: S2010/BMD-2402

-Universidad Autónoma de Madrid, Ministerio de Educación, Cultura y Deporte.

Grant Number: FPU13/00544 